\title{
Coupled quantum dots as quantum gates
}

\author{
Guido Burkard ${ }^{1 *}$, Daniel Loss ${ }^{1 \dagger}$, and David P. DiVincenzo ${ }^{2 \ddagger}$ \\ ${ }^{1}$ Department of Physics and Astronomy, University of Basel, \\ Klingelbergstrasse 82, CH-4056 Basel, Switzerland \\ ${ }^{2}$ IBM Research Division, T.J. Watson Research Center, \\ P.O. Box 218, Yorktown Heights, New York 10598
}

\begin{abstract}
We consider a new quantum gate mechanism based on electron spins in coupled semiconductor quantum dots. Such gates provide a general source of spin entanglement and can be used for quantum computers. We determine the exchange coupling $J$ in the effective Heisenberg model as a function of magnetic $(B)$ and electric fields, and of the inter-dot distance $a$ within the HeitlerLondon approximation of molecular physics. This result is refined by using sp-hybridization, and by the Hund-Mulliken molecular-orbit approach which leads to an extended Hubbard description for the two-dot system that shows a remarkable dependence on $B$ and $a$ due to the long-range Coulomb interaction. We find that the exchange $J$ changes sign at a finite field (leading to a pronounced jump in the magnetization) and then decays exponentially. The magnetization and the spin susceptibilities of the coupled dots are calculated. We show that the dephasing due to nuclear spins in GaAs can be strongly suppressed by dynamical nuclear spin polarization and/or by magnetic fields.
\end{abstract}

\section{INTRODUCTION}

Semiconductor quantum dots, sometimes referred to as artificial atoms, are small devices in which charge carriers are confined in all three dimensions [1]. The confinement is usually achieved by electrical gating and/or etching techniques applied e.g. to a two-dimensional electron gas (2DEG). Since the dimensions of quantum dots are on the order of the Fermi wavelength, their electronic spectrum consists of discrete energy levels which have been studied in great detail in conductance [1,2] and spectroscopy measurements [1, 3, 4 ]. In GaAs heterostructures the number of electrons in the dots can be changed one by one starting from zero [5]. Typical laboratory magnetic fields $(B \approx 1 \mathrm{~T})$ correspond to magnetic lengths on the order of $l_{B} \approx 10 \mathrm{~nm}$, being much larger than the Bohr radius of real atoms but of the same size as artificial atoms. As a consequence, the dot spectrum depends strongly on the applied magnetic field [1 3 ]. In coupled quantum dots which can be considered to some extent as artificial molecules, Coulomb blockade effects [6] and magnetization [7] have been observed as well as the formation of a delocalized "molecular state" [8].

Motivated by the rapid down-scaling of integrated circuits, there has been continued interest in classical logic devices made of electrostatically coupled quantum dots [9]. More recently, the discovery of new principles of computation based on quantum mechanics [10] has led to the idea of using coupled quantum dots for quantum computation [11]; many other proposed implementations have been explored, involving NMR 12 14], trapped ions [15], cavity QED [16], and Josephson junctions [17]. Solidstate devices open up the possibility of fabricating large integrated networks which would be required for realistic applications of quantum computers. A basic feature of the quantum-dot scenario [11] is to consider the electron spin $\mathbf{S}$ as the qubit (the qubit being the basic unit of information in the quantum computer). This stands in contrast to alternative proposals also based on quantum dots [18 21], in which it is the charge (orbital) degrees of freedom out of which a qubit is formed and represented in terms of a pseudospin-1/2. However, there are two immediate advantages of real spin over pseudospin: First, the qubit represented by a real spin- $1 / 2$ is always a well defined qubit; the two-dimensional Hilbert space is the entire space available, thus there are no extra dimensions into which the qubit state could "leak" [22]. Second, during a quantum computation phase coherence of the qubits must be preserved. It is thus an essential advantage of real spins that their dephasing times in GaAs can be on the order of microseconds [23], whereas for charge degrees of freedom dephasing times are typically much less, on the order of nanoseconds 24,25].

In addition to a well defined qubit, we also need a controllable "source of entanglement", i.e. a mechanism by which two specified qubits at a time can be entangled [26] so as to produce the fundamental quantum XOR (or controlled-NOT) gate operation, represented by a unitary operator $U_{\mathrm{XOR}}$ [27. This can be achieved by temporarily coupling two spins 11. As we will show in detail below, due to the Coulomb interaction and the Pauli exclusion principle the ground state of two coupled electrons is a spin singlet, i.e. a highly entangled spin state. This physical picture translates into an exchange coupling $J(t)$ between the two spins $\mathbf{S}_{1}$ and $\mathbf{S}_{2}$ described by a Heisenberg Hamiltonian

$$
H_{\mathrm{s}}(t)=J(t) \mathbf{S}_{1} \cdot \mathbf{S}_{2}
$$

If the exchange coupling is pulsed such that $\int d t J(t) / \hbar=$ $J_{0} \tau_{s} / \hbar=\pi(\bmod 2 \pi)$, the associated unitary time evolution $U(t)=T \exp \left(i \int_{0}^{t} H_{\mathrm{s}}(\tau) d \tau / \hbar\right)$ corresponds to the "swap" operator $U_{\text {sw }}$ which simply exchanges the quan- 
tum states of qubit 1 and $2 \sqrt{11}$. Furthermore, the quantum XOR can be obtained [11] by applying the sequence $\exp \left(i(\pi / 2) S_{1}^{z}\right) \exp \left(-i(\pi / 2) S_{2}^{z}\right) U_{\mathrm{sw}}^{1 / 2} \exp \left(i \pi S_{1}^{z}\right) U_{\mathrm{sw}}^{1 / 2} \equiv$ $U_{\mathrm{XOR}}$, i.e. a combination of "square-root of swap" $U_{\mathrm{sw}}^{1 / 2}$ and single-qubit rotations $\exp \left(i \pi S_{1}^{z}\right)$, etc. Since $U_{\mathrm{XOR}}$ (combined with single-qubit rotations) is proven to be a universal quantum gate 18,26, it can therefore be used to assemble any quantum algorithm. Thus, the study of a quantum XOR gate is essentially reduced to the study of the exchange mechanism and how the exchange coupling $J(t)$ can be controlled experimentally. We wish to emphasize that the switchable coupling mechanism described in the following need not be confined to quantum dots: the same principle can be applied to other systems, e.g. coupled atoms in a Bravais lattice, overlapping shallow donors in semiconductors such as $\mathrm{P}$ in $\mathrm{Si}$ [28], and so on. The main reason to concentrate here on quantum dots is that these systems are at the center of many ongoing experimental investigations in mesoscopic physics, and thus there seems to be reasonable hope that these systems can be made into quantum gates functioning along the lines proposed here.

In view of this motivation we study in the following the spin dynamics of two laterally coupled quantum dots containing a single electron each. We show that the exchange coupling $J(B, E, a)$ can be controlled by a magnetic field $B$ (leading to wave function compression), or by an electric field $E$ (leading to level detuning), or by varying the barrier height or equivalently the inter-dot distance $2 a$ (leading to a suppression of tunneling between the dots). The dependence on these parameters is of direct practical interest, since it opens the door to tailoring the exchange $J(t)$ for the specific purpose of creating quantum gates. We further calculate the static and dynamical magnetization responses in the presence of perpendicular and parallel magnetic fields, and show that they give experimentally accessible information about the exchange $J$. Our analysis is based on an adaptation of HeitlerLondon and Hund-Mulliken variational techniques [29] to parabolically confined coupled quantum dots. In particular, we present an extension of the Hubbard approximation induced by the long-range Coulomb interaction. We find a striking dependence of the Hubbard parameters on the magnetic field and inter-dot distance which is of relevance also for atomic-scale Hubbard physics in the presence of long-range Coulomb interactions. Finally, we discuss the effects of dephasing induced by nuclear spins in GaAs and show that dephasing can be strongly reduced by dynamically polarizing the nuclear spins and/or by magnetic fields.

The paper is organized as follows. In Sec. II we introduce the model for the quantum gate in terms of coupled dots. In Sec. III we calculate the exchange coupling first in the Heitler-London and then in the Hund-Mulliken approach. There we also discuss the Hubbard limit and the new features arising from the long range nature of the Coulomb interactions. In Sec. IV we consider the effects of imperfections leading to dephasing and gate errors; in particular, we consider dephasing resulting from nuclear spins in GaAs. Implications for experiments on magnetization and spin susceptibilities are presented in Sec. V, and Sec. VI contains some concluding remarks on networks of gates with some suggestions for singlequbit gates operated by local magnetic fields. Finally, we mention that a preliminary account of some of the results presented here has been given in Ref. [30].

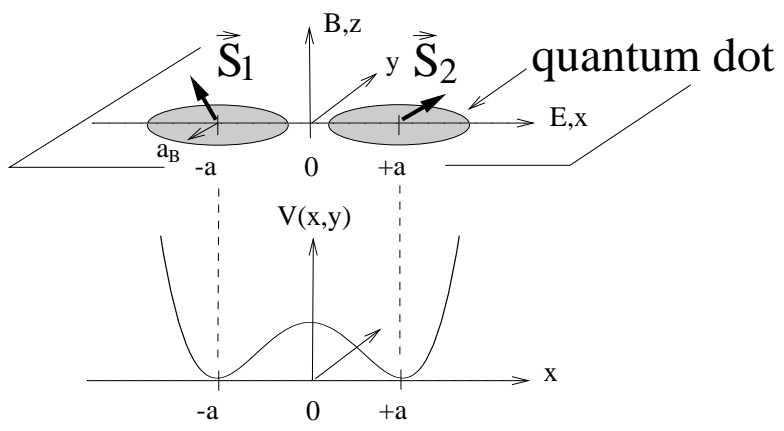

FIG. 1. Two coupled quantum dots with one valence electron per dot. Each electron is confined to the $x y$ plane. The spins of the electrons in dots 1 and 2 are denoted by $\mathbf{S}_{1}$ and $\mathbf{S}_{2}$. The magnetic field $B$ is perpendicular to the plane, i.e. along the $z$ axis, and the electric field $E$ is in-plane and along the $x$ axis. The quartic potential is given in Eq. (3) and is used to model the coupling of two harmonic wells centered at $( \pm a, 0,0)$. The exchange coupling $J$ between the spins is a function of $B, E$, and the inter-dot distance $2 a$.

\section{MODEL FOR THE QUANTUM GATE}

We consider a system of two laterally coupled quantum dots containing one (conduction band) electron each, see Fig. 11. It is essential that the electrons are allowed to tunnel between the dots, and that the total wave function of the coupled system must be antisymmetric. It is this fact which introduces correlations between the spins via the charge (orbital) degrees of freedom. For definiteness we shall use in the following the parameter values recently determined for single GaAs heterostructure quantum dots [5] that are formed in a 2DEG; this choice is not crucial for the following analysis but it allows us to illustrate our analytical results with realistic numbers. The Hamiltonian for the coupled system is then given by

$$
\begin{aligned}
H & =\sum_{i=1,2} h_{i}+C+H_{\mathrm{Z}}=H_{\mathrm{orb}}+H_{\mathrm{Z}} \\
h_{i} & =\frac{1}{2 m}\left(\mathbf{p}_{i}-\frac{e}{c} \mathbf{A}\left(\mathbf{r}_{i}\right)\right)^{2}+e x_{i} E+V\left(\mathbf{r}_{i}\right), \\
C & =\frac{e^{2}}{\kappa\left|\mathbf{r}_{1}-\mathbf{r}_{2}\right|} .
\end{aligned}
$$

The single-particle Hamiltonian $h_{i}$ describes the electron dynamics confined to the $x y$-plane. The electrons have 
an effective mass $m\left(m=0.067 m_{e}\right.$ in GaAs $)$ and carry a spin-1/2 $\mathbf{S}_{i}$. The dielectric constant in GaAs is $\kappa=$ 13.1. We allow for a magnetic field $\mathbf{B}=(0,0, B)$ applied along the $z$-axis and which couples to the electron charge via the vector potential $\mathbf{A}(\mathbf{r})=\frac{B}{2}(-y, x, 0)$. We also allow for an electric field $E$ applied in-plane along the xdirection, i.e. along the line connecting the centers of the dots. The coupling of the dots (which includes tunneling) is modeled by a quartic potential,

$$
V(x, y)=\frac{m \omega_{0}^{2}}{2}\left(\frac{1}{4 a^{2}}\left(x^{2}-a^{2}\right)^{2}+y^{2}\right),
$$

which separates (for $x$ around $\pm a$ ) into two harmonic wells of frequency $\omega_{0}$, one for each dot, in the limit of large inter-dot distance, i.e. for $2 a \gg 2 a_{\mathrm{B}}$, where $a$ is half the distance between the centers of the dots, and $a_{\mathrm{B}}=\sqrt{\hbar / m \omega_{0}}$ is the effective Bohr radius of a single isolated harmonic well. This choice for the potential is motivated by the experimental fact [5] that the spectrum of single dots in GaAs is well described by a parabolic confinement potential, e.g. with $\hbar \omega_{0}=3 \mathrm{meV}$ [5]. We note that increasing (decreasing) the inter-dot distance is physically equivalent to raising (lowering) the inter-dot barrier, which can be achieved experimentally by e.g. applying a gate voltage between the dots [6]. Thus, the effect of such gate voltages is described in our model simply by a change of the inter-dot distance $2 a$. We also note that it is only for simplicity that we choose the two dots to be exactly identical, no qualitative changes will occur in the following analysis if the dots are only approximately equal and approximately of parabolic shape.

The (bare) Coulomb interaction between the two electrons is described by $C$. The screening length $\lambda$ in almost depleted regions like few-electron quantum dots can be expected to be much larger than the bulk 2DEG screening length (which is about $40 \mathrm{~nm}$ in GaAs). Therefore, $\lambda$ is large compared to the size of the coupled system, $\lambda \gg 2 a \approx 40 \mathrm{~nm}$ for small dots, and we will consider the limit of unscreened Coulomb interaction $(\lambda / a \gg 1)$ throughout this work.

The magnetic field $B$ also couples to the electron spins via the Zeeman term $H_{\mathrm{Z}}=g \mu_{\mathrm{B}} \sum_{i} \mathbf{B}_{i} \cdot \mathbf{S}_{i}$, where $g$ is the effective g-factor ( $g \approx-0.44$ for GaAs), and $\mu_{\mathrm{B}}$ the Bohr magneton. The ratio between the Zeeman splitting and the relevant orbital energies is small for all $B$ values of interest here; indeed, $g \mu_{\mathrm{B}} B / \hbar \omega_{0} \lesssim 0.03$, for $B \ll B_{0}=\left(\hbar \omega_{0} / \mu_{\mathrm{B}}\right)\left(m / m_{e}\right) \approx 3.5 \mathrm{~T}$, and $g \mu_{\mathrm{B}} B / \hbar \omega_{\mathrm{L}} \lesssim$ 0.03 , for $B \gg B_{0}$, where $\omega_{\mathrm{L}}=e B / 2 m c$ is the Larmor frequency, and where we used $\hbar \omega_{0}=3 \mathrm{meV}$. Thus, we can safely ignore the Zeeman splitting when we discuss the orbital degrees of freedom and include it later into the effective spin Hamiltonian. Also, in the fewelectron system we are dealing with, spin-orbit effects can be completely neglected since $H_{\mathrm{so}} / \hbar \omega_{0} \approx 10^{-7}$, where $H_{\mathrm{so}}=\left(\omega_{0}^{2} / 2 m c^{2}\right) \mathbf{L} \cdot \mathbf{S}$ is the spin-orbit coupling of an electron in a parabolic confinement potential 30]. This has the important implication that dephasing effects induced e.g. by potential or charge fluctuations in the surroundings of the isolated dots can couple only to the charge of the electron so that they have very small influence on the phase coherence of the isolated spin itself (for dephasing induced by coupling the dots see Sec. IV). It is for this reason that it is preferable to consider dots containing electrons instead of holes, since holes will typically have a sizable spin-orbit interaction [1].

Finally, we assume a low-temperature description where $k T \ll \hbar \omega_{0}$, so that we can restrict ourselves to the two lowest orbital eigenstates of $H_{\mathrm{orb}}$, one of which is symmetric (spin singlet) and the other one antisymmetric (spin triplet). In this reduced (four-dimensional) Hilbert space, $H_{\text {orb }}$ can be replaced by the effective Heisenberg spin Hamiltonian Eq. (1),$H_{\mathrm{s}}=J \mathbf{S}_{1} \cdot \mathbf{S}_{2}$, where the exchange energy $J=\epsilon_{\mathrm{t}}-\epsilon_{\mathrm{s}}$ is the difference between the triplet and singlet energy which we wish to calculate. The above model cannot be solved in an analytically closed form. However, the analogy between atoms and quantum dots (artificial atoms) provides us with a powerful set of variational methods from molecular physics for finding $\epsilon_{\mathrm{t}}$ and $\epsilon_{\mathrm{s}}$. Note that the typical energy scale $\hbar \omega_{0} \approx \mathrm{meV}$ in our quantum dot is about a thousand times smaller than the energies $(\mathrm{Ry} \approx \mathrm{eV})$ in a hydrogen atom, whereas the quantum dot is larger by about the same factor. This is important because their size makes quantum dots much more susceptible to magnetic fields than atoms. In analogy to atomic physics, we call the size of the electron orbitals in a quantum dot the Bohr radius, although it is determined by the confining potential rather than by the Coulomb attraction to a positively charged nucleus. For harmonic confinement $a_{\mathrm{B}}=\sqrt{\hbar / m \omega_{0}}$ is about $20 \mathrm{~nm}$ for $\hbar \omega_{0}=3 \mathrm{meV}$.

\section{EXCHANGE ENERGY}

\section{A. Heitler-London approach}

We consider first the Heitler-London approximation, and then refine this approach by including hybridization as well as double occupancy in a Hund-Mulliken approach, which will finally lead us to an extension of the Hubbard description. We will see, however, that the qualitative features of $J$ as a function of the control parameters are already captured by the simplest Heitler-London approximation for the artificial hydrogen molecule described by Eq. 2. In this approximation, one starts from single-dot ground-state orbital wavefunctions $\varphi(\mathbf{r})$ and combines them into the (anti-) symmetric twoparticle orbital state vector

$$
\left|\Psi_{ \pm}\right\rangle=\frac{|12\rangle \pm|21\rangle}{\sqrt{2\left(1 \pm S^{2}\right)}}
$$

the positive (negative) sign corresponding to the spin singlet (triplet) state, and $S=\int d^{2} r \varphi_{+a}^{*}(\mathbf{r}) \varphi_{-a}(\mathbf{r})=\langle 2 \mid 1\rangle$ 
denoting the overlap of the right and left orbitals. A nonvanishing overlap implies that the electrons tunnel between the dots (see also Sec. III B). Here, $\varphi_{-a}(\mathbf{r})=\langle\mathbf{r} \mid 1\rangle$ and $\varphi_{+a}(\mathbf{r})=\langle\mathbf{r} \mid 2\rangle$ denote the one-particle orbitals centered at $\mathbf{r}=(\mp a, 0)$, and $|i j\rangle=|i\rangle|j\rangle$ are two-particle product states. The exchange energy is then obtained through $J=\epsilon_{\mathrm{t}}-\epsilon_{\mathrm{s}}=\left\langle\Psi_{-}\left|H_{\text {orb }}\right| \Psi_{-}\right\rangle-\left\langle\Psi_{+}\left|H_{\text {orb }}\right| \Psi_{+}\right\rangle$. The single-dot orbitals for harmonic confinement in two dimensions in a perpendicular magnetic field are the Fock-Darwin states [31], which are the usual harmonic oscillator states, magnetically compressed by a factor $b=\omega / \omega_{0}=\sqrt{1+\omega_{\mathrm{L}}^{2} / \omega_{0}^{2}}$, where $\omega_{\mathrm{L}}=e B / 2 m c$ denotes the Larmor frequency. The ground state (energy $\left.\hbar \omega=b \hbar \omega_{0}\right)$ centered at the origin is

$$
\varphi(x, y)=\sqrt{\frac{m \omega}{\pi \hbar}} e^{-m \omega\left(x^{2}+y^{2}\right) / 2 \hbar} .
$$

Shifting the single particle orbitals to $( \pm a, 0)$ in the presence of a magnetic field we obtain $\varphi_{ \pm a}(x, y)=$ $\exp \left( \pm i y a / 2 l_{B}^{2}\right) \varphi(x \mp a, y)$. The phase factor involving the magnetic length $l_{B}=\sqrt{\hbar c / e B}$ is due to the gauge transformation $\mathbf{A}_{ \pm a}=B(-y, x \mp a, 0) / 2 \rightarrow \mathbf{A}=$ $B(-y, x, 0) / 2$. The matrix elements of $H_{\text {orb }}$ needed to calculate $J$ are found by adding and subtracting the harmonic potential centered at $x=-(+) a$ for electron 1(2) in $H_{\text {orb }}$, which then takes the form $H_{\text {orb }}=$ $h_{-a}^{0}\left(\mathbf{r}_{1}\right)+h_{+a}^{0}\left(\mathbf{r}_{2}\right)+W+C$, where $h_{ \pm a}^{0}\left(\mathbf{r}_{i}\right)=\left(\mathbf{p}_{i}-\right.$ $\left.e \mathbf{A}\left(\mathbf{r}_{i}\right) / c\right)^{2} / 2 m+m \omega^{2}\left(\left(x_{i} \mp a\right)^{2}+y_{i}^{2}\right) / 2$ is the FockDarwin Hamiltonian shifted to $( \pm a, 0)$, and $W(x, y)=$ $V(x, y)-m \omega^{2}\left(\left(x_{1}+a\right)^{2}+\left(x_{2}-a\right)^{2}\right) / 2$. We obtain

$$
J=\frac{2 S^{2}}{1-S^{4}}\left(\langle 12|C+W| 12\rangle-\frac{\operatorname{Re}\langle 12|C+W| 21\rangle}{S^{2}}\right),
$$

where the overlap becomes $S=\exp \left(-m \omega a^{2} / \hbar-\right.$ $\left.a^{2} \hbar / 4 l_{B}^{4} m \omega\right)$. Evaluation of the matrix elements of $C$ and $W$ yields (see also [30])

$$
\begin{aligned}
J & =\frac{\hbar \omega_{0}}{\sinh \left(2 d^{2}(2 b-1 / b)\right)}\left[c \sqrt { b } \left(e^{-b d^{2}} \mathrm{I}_{0}\left(b d^{2}\right)\right.\right. \\
& \left.\left.-e^{d^{2}(b-1 / b)} \mathrm{I}_{0}\left(d^{2}(b-1 / b)\right)\right)+\frac{3}{4 b}\left(1+b d^{2}\right)\right],
\end{aligned}
$$

where we introduce the dimensionless distance $d=a / a_{\mathrm{B}}$, and $\mathrm{I}_{0}$ is the zeroth order Bessel function. The first and second terms in Eq. (7) are due to the Coulomb interaction $C$, where the exchange term enters with a minus sign. The parameter $c=\sqrt{\pi / 2}\left(e^{2} / \kappa a_{\mathrm{B}}\right) / \hbar \omega_{0}(\approx 2.4$, for $\hbar \omega_{0}=3 \mathrm{meV}$ ) is the ratio between Coulomb and confining energy. The last term comes from the confinement potential $W$. The result $J(B)$ is plotted in Fig. 2 (dashed line). Note that typically $\left|J / \hbar \omega_{0}\right| \lesssim 0.2$. Also, we see that $J>0$ for $B=0$, which must be the case for a two-particle system that is time-reversal invariant [29].

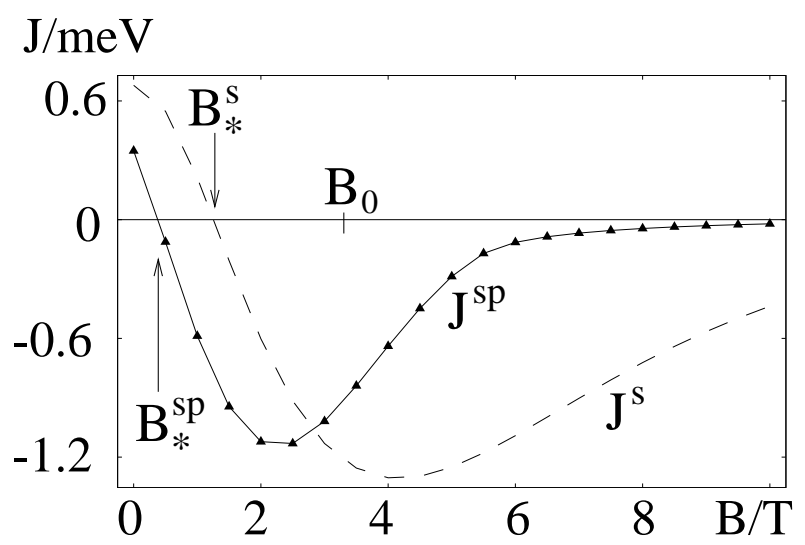

FIG. 2. Exchange energy $J$ in units of meV plotted against the magnetic field $B$ (in units of Tesla), as obtained from the s-wave Heitler-London approximation (dashed line), Eq. (耳), and the result from the improved sp-hybridized Heitler-London approximation (triangles) which is obtained numerically as explained in the text. Note that the qualitative behavior of the two curves is similar, i.e. they both have zeroes, the s-wave approximation at $B_{*}^{\mathrm{s}}$, and the sp-hybridized approximation at $B_{*}^{\mathrm{sp}}$, and also both curves vanish exponentially for large fields. $B_{0}=\left(\hbar \omega_{0} / \mu_{\mathrm{B}}\right)\left(\mathrm{m} / \mathrm{m}_{e}\right)$ denotes the crossover field to magnetically dominated confining $\left(B \gg B_{0}\right)$. The curves are given for a confinement energy $\hbar \omega_{0}=3 \mathrm{meV}$ (implying for the Coulomb parameter $c=2.42$ ), and inter-dot distance $a=0.7 a_{\mathrm{B}}$.

The most remarkable feature of $J(B)$, however, is the change of sign from positive to negative at $B=B_{*}^{\mathrm{s}}$, which occurs over a wide range of parameters $c$ and $a$. This singlet-triplet crossing occurs at about $B_{*}^{\mathrm{s}}=1.3 \mathrm{~T}$ for $\hbar \omega_{0}=3 \mathrm{meV}(c=2.42)$ and $d=0.7$. The transition from antiferromagnetic $(J>0)$ to ferromagnetic $(J<0)$ spin-spin coupling with increasing magnetic field is caused by the long-range Coulomb interaction, in particular by the negative exchange term, the second term in Eq. (7). As $B \gg B_{0}\left(\approx 3.5 \mathrm{~T}\right.$ for $\left.\hbar \omega_{0}=3 \mathrm{meV}\right)$, the magnetic field compresses the orbits by a factor $b \approx B / B_{0} \gg 1$ and thereby reduces the overlap of the wavefunctions, $S^{2} \approx \exp \left(-2 d^{2}(2 b-1 / b)\right)$, exponentially strongly. Similarly, the overlap decays exponentially for large inter-dot distances, $d \gg 1$. Note however, that this exponential suppression is partly compensated by the exponentially growing exchange term $\langle 12|C| 21\rangle / S^{2} \propto \exp \left(2 d^{2}(b-1 / b)\right)$. As a result, the exchange coupling $J$ decays exponentially as $\exp \left(-2 d^{2} b\right)$ for large $b$ or $d$, as shown in Fig. $3 \mathrm{~b}$ for $B=0(b=1)$. Thus, the exchange coupling $J$ can be tuned through zero and then suppressed to zero by a magnetic field in a very efficient way. We note that our Heitler-London approximation breaks down explicitly (i.e. $J$ becomes negative even when $B=0$ ) for certain inter-dot distances when $c$ exceeds 2.8. Finally, a similar singlet-triplet crossing as function of the magnetic field has been found in single dots with two electrons [32]. 
The exchange energy $J$ also depends on the applied electric field $E$. The additional term $e\left(x_{1}+x_{2}\right) E$ in the potential merely shifts the one-particle orbitals by $\Delta x=e E / m \omega_{0}^{2}$, raising the energy of both the singlet and triplet states. Since the singlet energy turns out to be less affected by this shift than the triplet, the exchange energy $J$ increases with increasing $E$,

$J(B, E)=J(B, 0)+\frac{\hbar \omega_{0}}{\sinh \left(2 d^{2}(2 b-1 / b)\right)} \frac{3}{2} \frac{1}{d^{2}}\left(\frac{e E a}{\hbar \omega_{0}}\right)^{2}$,

the increase being proportional to $m \omega_{0}^{2}(\Delta x)^{2}$. [We note that this increase of $J(B, E)$ is qualitatively consistent with what one finds from a standard two-level approximation of a $1 \mathrm{D}$ double-well potential (with $J(B, 0)$ being the effective tunnel splitting) in the presence of a bias given by $e E a$.] The variational Ansatz leading to Eq. (8) is expected to remain accurate as long as $J(B, E)-J(B, 0) \lesssim J(B, 0)$; for larger $E$-fields the levels of the dots get completely detuned and the overlap of the wavefunctions (i.e. the coherent tunneling) between the dots is suppressed. Of course, a sufficiently large electric field will eventually force both electrons on to the same dot, which is the case when $e E a$ exceeds the onsite repulsion $U(\gg J(B, E=0)$, see below). However, this situation, which would correspond to a quantum dot helium [33], is not of interest in the present context. Conversely, in case of dots of different size (or shape) where the energy levels need not be aligned a priori, an appropriate electric field can be used to match the levels of the two dots, thus allowing coherent tunneling even in those systems. Recent conductance measurements [8] on coupled dots of different size (containing several electrons) with electrostatic tuning have revealed clear evidence for a delocalized molecular state.

A shortcoming of the simple approximation described above is that solely ground-state single-particle orbitals were taken into account and mixing with excited oneparticle states due to interaction is neglected. This approximation is self-consistent if $J \ll \Delta \epsilon$, where $\Delta \epsilon$ denotes the single-particle level separation between the ground state and the first excited state. We find $|J / \Delta \epsilon|<$ 0.25 at low fields $B \leq 1.75 \mathrm{~T}$, therefore $J(B)$ is at least qualitatively correct in this regime. At higher fields $|J / \Delta \epsilon| \approx 1$, indicating substantial mixing with higher orbitals. An improved Heitler-London variational Ansatz is obtained by introducing sp-hybridized single-dot orbitals (in analogy to molecular physics), i.e. $\phi=\varphi_{\mathrm{s}}+\alpha \varphi_{\mathrm{p} x}+$ $i \beta \varphi_{\mathrm{p} y}$, where $\varphi_{\mathrm{s}}=\varphi$ is the s-orbital introduced above, $\varphi_{\mathrm{p} q}=\sqrt{\frac{2}{\pi}} m \omega q \exp \left(-m \omega r^{2} / 2 \hbar\right) / \hbar, q=x, y$, are the lowest two Fock-Darwin excited states (at zero field) with angular momentum $|\ell|=1$, and $\alpha$ and $\beta$ are real variational parameters to be determined by minimization of the singlet and triplet energies $\epsilon_{\mathrm{s}, \mathrm{t}}(\alpha, \beta)$, which is done numerically. The $\varphi_{\mathrm{p} q}$ are chosen to be real, they are however not eigenstates of the single-particle Hamiltonian, which are $\varphi_{\mathrm{p} x} \pm i \varphi_{\mathrm{p} y}$ (with eigenenergy $2 \hbar \omega \pm \hbar \omega_{\mathrm{L}}$ ). Note that while $\epsilon_{\mathrm{s}, \mathrm{t}}$ decrease only by $\approx 1 \%$ due to hybridization, the relative variation of $J=\epsilon_{\mathrm{t}}-\epsilon_{\mathrm{s}}$ can still be substantial. Nevertheless, the resulting exchange energy $J^{\text {sp }}$ (Fig. 2) is only quantitatively different from the pure s-wave result $J \equiv J^{\mathrm{s}}$, Eq. (7). At low fields, $J^{\mathrm{sp}}<J^{\mathrm{s}}$ and the change of sign occurs already at about $B_{*}^{\mathrm{sp}} \simeq 0.4 \mathrm{~T}<B_{*}^{\mathrm{s}}$. At high fields, $J^{\text {sp }}$ shows a much more pronounced decay as a function of $B$.

Being a completely orbital effect, the exchange interaction between spins of course competes with the Zeeman coupling $H_{Z}$ of the spins to the magnetic field. In our case, however, the Zeeman energy $H_{Z}$ is small and exceeds the exchange energy (polarizing the spins) only in a narrow window (about $0.1 \mathrm{~T}$ wide) around $B_{*}^{\mathrm{sp}}$ and again for high fields $(B>4 \mathrm{~T})$.

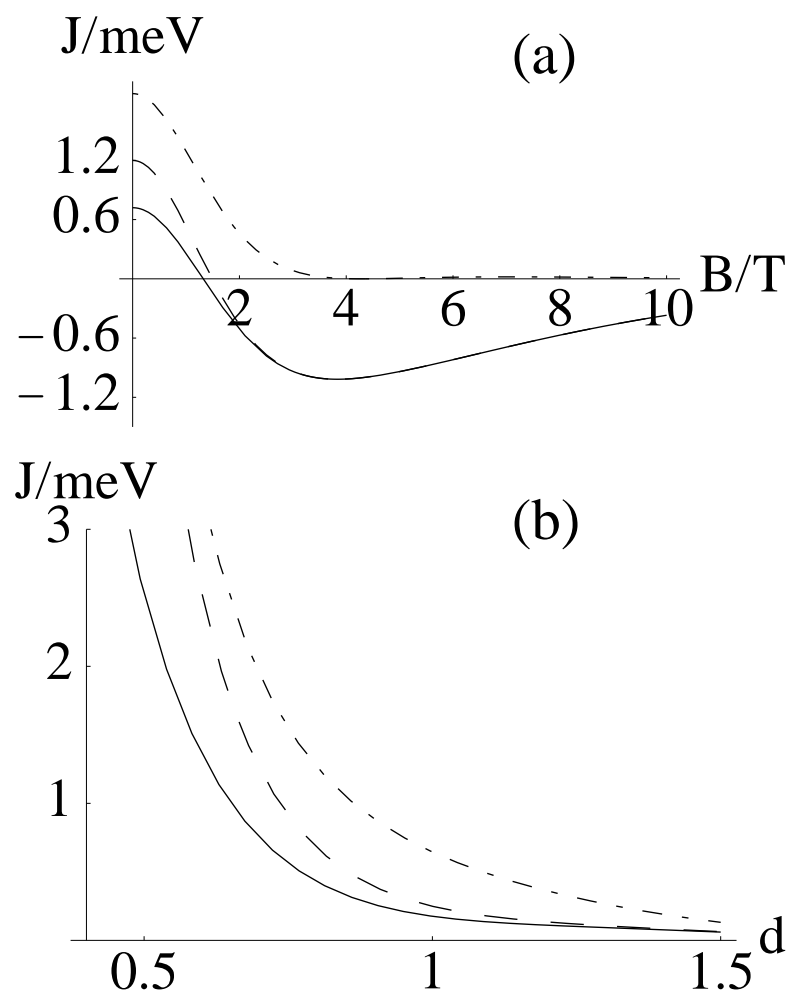

FIG. 3. The exchange coupling $J$ obtained from Hund-Mulliken (full line), Eq. (11), and from the extended Hubbard approximation (dashed line), Eq. (12). For comparison, we also plot the usual Hubbard approximation where the long-range interaction term $V$ is omitted, i.e. $J=4 t_{\mathrm{H}}^{2} / U_{\mathrm{H}}$ (dashed-dotted line). In (a), $J$ is plotted as a function of the magnetic field $B$ at fixed inter-dot distance $\left(d=a / a_{\mathrm{B}}=0.7\right)$, and for $c=2.42$, in (b) as a function of inter-dot distance $d=a / a_{\mathrm{B}}$ at zero field $(B=0)$, and again $c=2.42$. For these parameter values, the s-wave Heitler-London $J$, Eq. (7), and the Hund-Mulliken $J$ (full line) are almost identical. 


\section{B. Hund-Mulliken approach and Hubbard Limit}

We turn now to the Hund-Mulliken method of molecular orbits 29 which extends the Heitler-London approach by including also the two doubly occupied states, which both are spin singlets. This extends the orbital Hilbert space from two to four dimensions. First, the single particle states have to be orthonormalized, leading to the states $\Phi_{ \pm a}=\left(\varphi_{ \pm a}-g \varphi_{\mp a}\right) / \sqrt{1-2 S g+g^{2}}$, where $S$ again denotes the overlap of $\varphi_{-a}$ with $\varphi_{+a}$ and $g=\left(1-\sqrt{1-S^{2}}\right) / S$. Then, diagonalization of

$$
H_{\text {orb }}=2 \epsilon+\left(\begin{array}{cccc}
U & X & -\sqrt{2} t_{\mathrm{H}} & 0 \\
X & U & -\sqrt{2} t_{\mathrm{H}} & 0 \\
-\sqrt{2} t_{\mathrm{H}} & -\sqrt{2} t_{\mathrm{H}} & V_{+} & 0 \\
0 & 0 & 0 & V_{-}
\end{array}\right)
$$

in the space spanned by $\Psi_{ \pm a}^{\mathrm{d}}\left(\mathbf{r}_{1}, \mathbf{r}_{2}\right)=\Phi_{ \pm a}\left(\mathbf{r}_{1}\right) \Phi_{ \pm a}\left(\mathbf{r}_{2}\right)$, $\Psi_{ \pm}^{\mathrm{s}}\left(\mathbf{r}_{1}, \mathbf{r}_{2}\right)=\left[\Phi_{+a}\left(\mathbf{r}_{1}\right) \Phi_{-a}\left(\mathbf{r}_{2}\right) \pm \Phi_{-a}\left(\mathbf{r}_{1}\right) \Phi_{+a}\left(\mathbf{r}_{2}\right)\right] / \sqrt{2}$ yields the eigenvalues $\epsilon_{\mathrm{s} \pm}=2 \epsilon+U_{\mathrm{H}} / 2+V_{+} \pm$ $\sqrt{U_{\mathrm{H}}^{2} / 4+4 t_{\mathrm{H}}^{2}}, \epsilon_{\mathrm{s} 0}=2 \epsilon+U_{\mathrm{H}}-2 X+V_{+}$(singlet), and $\epsilon_{\mathrm{t}}=2 \epsilon+V_{-}$(triplet), where the quantities

$$
\begin{aligned}
\epsilon & =\left\langle\Phi_{ \pm a}\left|h_{ \pm a}^{0}\right| \Phi_{ \pm a}\right\rangle, \\
t_{\mathrm{H}} & =t-w=\left\langle\Phi_{ \pm a}\left|h_{ \pm}^{0}\right| \Phi_{\mp a}\right\rangle-\left\langle\Psi_{+}^{\mathrm{s}}|C| \Psi_{ \pm a}^{\mathrm{d}}\right\rangle / \sqrt{2}, \\
V & =V_{-}-V_{+}=\left\langle\Psi_{-}^{\mathrm{s}}|C| \Psi_{-}^{\mathrm{s}}\right\rangle-\left\langle\Psi_{+}^{\mathrm{s}}|C| \Psi_{+}^{\mathrm{s}}\right\rangle, \\
X & =\left\langle\Psi_{ \pm a}^{\mathrm{d}}|C| \Psi_{\mp a}^{\mathrm{d}}\right\rangle, \\
U_{\mathrm{H}} & =U-V_{+}+X \\
& =\left\langle\Psi_{ \pm a}^{\mathrm{d}}|C| \Psi_{ \pm a}^{\mathrm{d}}\right\rangle-\left\langle\Psi_{+}^{\mathrm{s}}|C| \Psi_{+}^{\mathrm{s}}\right\rangle+\left\langle\Psi_{ \pm a}^{\mathrm{d}}|C| \Psi_{\mp a}^{\mathrm{d}}\right\rangle,
\end{aligned}
$$

all depend on the magnetic field $B$. The exchange energy is the gap between the lowest singlet and the triplet state

$$
J=\epsilon_{\mathrm{t}}-\epsilon_{\mathrm{s}-}=V-\frac{U_{\mathrm{H}}}{2}+\frac{1}{2} \sqrt{U_{\mathrm{H}}^{2}+16 t_{\mathrm{H}}^{2}} .
$$

In the standard Hubbard approach for short-range Coulomb interactions (and without $B$-field) [29] $J$ reduces to $-U / 2+\sqrt{U^{2}+16 t^{2}} / 2$, where $t$ denotes the hopping matrix element, and $U$ the on-site repulsion (cf. Eq. (10)). Thus, $t_{\mathrm{H}}$ and $U_{\mathrm{H}}$ are the extended hopping matrix element and the on-site repulsion, resp., renormalized by long-range Coulomb interactions. The remaining two singlet energies $\epsilon_{\mathrm{S}+}$ and $\epsilon_{\mathrm{s} 0}$ are separated from $\epsilon_{\mathrm{t}}$ and $\epsilon_{\mathrm{s}-}$ by a gap of order $U_{\mathrm{H}}$ and are therefore neglected for the study of low-energy properties. The evaluation of the matrix elements is straightforward but lengthy, and we give the results in Appendix A. Typically, the "Hubbard ratio" $t_{\mathrm{H}} / U_{\mathrm{H}}$ is less than 1 , e.g., if $d=0.7, \hbar \omega_{0}=3 \mathrm{meV}$, and $B=0$, we obtain $t_{\mathrm{H}} / U_{\mathrm{H}}=0.34$, and it decreases with increasing $B$. Therefore, we are in an extended Hubbard limit, where $J$ takes the form

$$
J=\frac{4 t_{\mathrm{H}}^{2}}{U_{\mathrm{H}}}+V .
$$

The first term has the form of the standard Hubbard approximation [35] (invoked previously [11]) but with $t_{\mathrm{H}}$ and $U_{\mathrm{H}}$ being renormalized by long-range Coulomb interactions. The second term $V$ is new and accounts for the difference in Coulomb energy between the singly occupied singlet and triplet states $\Psi_{ \pm}^{\mathrm{s}}$. It is precisely this $V$ that makes $J$ negative for high magnetic fields, whereas $t_{\mathrm{H}}^{2} / U_{\mathrm{H}}>0$ for all values of $B$ (see Fig. 3a). Thus, the usual Hubbard approximation (i.e. without $V$ ) would not give reliable results, neither for the $B$-dependence (Fig. 3a) nor for the dependence on the inter-dot distance $a$ (Fig. 3b) [36]. Since only the singlet space has been enlarged, it is clear that we obtain a lower singlet energy $\epsilon_{\mathrm{s}}$ than that from the s-wave Heitler-London calculation, but the same triplet energy $\epsilon_{\mathrm{t}}$, and therefore $J=\epsilon_{\mathrm{t}}-\epsilon_{\mathrm{s}}$ exceeds the s-wave Heitler-London result, Eq. (7). However, the on-site Coulomb repulsion $U \propto c$ strongly suppresses the doubly occupied states $\Psi_{ \pm a}^{\mathrm{d}}$ and already for the value of $c=2.4$ (corresponding to $\hbar \omega_{0}=3 \mathrm{meV}$ ) we obtain almost perfect agreement with the s-wave HeitlerLondon result (Fig. 2). For large fields, i.e. $B \gg B_{0}$, the suppression becomes even stronger $(U \propto \sqrt{B})$ because the electron orbits become compressed with increasing $B$ and two electrons on the same dot are confined to a smaller area leading to an increased Coulomb energy.

\section{DEPHASING AND QUANTUM GATE ERRORS}

We allow now for imperfections and discuss first the dephasing resulting from coupling to the environment, and then address briefly the issue of errors during the quantum-gate operation. We have already pointed out that dephasing in the charge sector will have little effect on the (uncoupled) spins due to the smallness of the spin-orbit interaction. Similarly, the dipolar interaction between the qubit spin and the surrounding spins is also minute, it can be estimated as $\left(g \mu_{\mathrm{B}}\right)^{2} / a_{\mathrm{B}}^{3} \approx 10^{-9}$ meV. Although both couplings are extremely small they will eventually lead to dephasing for sufficiently long times. We have described such weak-coupling dephasing in terms of a reduced master equation elsewhere [1], and we refer the interested reader to this work. Since this type of dephasing is small it can be eliminated by error correction schemes [37.

Next, we consider the dephasing due to nuclear spins in GaAs semiconductors, where both $\mathrm{Ga}$ and As possess a nuclear spin $I=3 / 2$. There is a sizable hyperfine coupling between the electron-spin $(s=1 / 2)$ and all the nuclear spins in the quantum dot which might easily lead to a flip of the electron spin and thus cause an error in the quantum computation. We shall now estimate this effect and show that it can be substantially reduced by spin polarization or by a field. We consider an electron spin $\mathbf{S}$ in contact with $N$ nuclear spins $\mathbf{I}^{(i)}$ in the presence of a magnetic field $B \| z$. The corresponding Hamiltonian is given by $H=A \mathbf{S} \cdot \mathbf{I}+b_{z} S_{z}+\tilde{b}_{z} I_{z}=H_{0}+V$, where 


$$
H_{0}=A S_{z} I_{z}+b_{z} S_{z}+\tilde{b}_{z} I_{z}, V=A\left(S_{+} I_{-}+S_{-} I_{+}\right) / 2
$$

Here, $A$ is a hyperfine coupling, $\mathbf{I}=\sum_{i=1}^{N} \mathbf{I}^{(i)}$ is the total nuclear spin, and $b_{z}=g \mu_{\mathrm{B}} B_{z}, \tilde{b}_{z}=g_{N} \mu_{N} B_{z}$ ( $g_{N}$ and $\mu_{N}$ denote the nuclear $\mathrm{g}$ factor and magneton). Consider the initial eigenstate $|i\rangle$ of $H_{0}$, which we will consider to be one basis vector for the qubit, where the electron spin is up (in the $S_{z}$ basis), and the nuclear spins are in a product state of $I_{z}^{(i)}$-eigenstates with total $I_{z}=p N I(-1 \leq p \leq 1)$, i.e. in a state with polarization $p$ along the $z$-axis; here, $p= \pm 1$ means that the nuclear spins are fully polarized in positive (negative) $z$-direction, and $p=0$ means no polarization. Due to the hyperfine coupling the electron spin can flip (i.e. dephase) with the entire system going into a final state $|k\rangle$ which is again a product state but now with the electronspin down, and, due to conservation of total spin, the $z$-component $I_{z}^{(k)}$ of one and only one nuclear spin having increased by $2 s=1$. All final states $|k\rangle$ are degenerate and again eigenstates of $H_{0}$ with eigenenergy $E_{f}$. We will consider this process now within time-dependent perturbation theory and up to second order in $V$. The energy difference between initial and final states amounts to $E_{i}-E_{f} \approx 2 s\left[A(p I N+s)+b_{z}\right]$, where we use that $b_{z} \gg \tilde{b}_{z}$. For the reversed process with an electronspin flip from down to up but with the same initial polarization for the nuclear spins the energy difference is $\approx-2 s\left[A(p I N-s)+b_{z}\right]$. The total transition probability to leave the initial state $|i\rangle$ after time $t$ has elapsed is then

$$
P_{i}(t)=\left(\frac{2 \sin \left(\left(E_{f}-E_{i}\right) t / 2 \hbar\right)}{E_{f}-E_{i}}\right)^{2} \sum_{k(\neq i)}|\langle k|V| i\rangle|^{2} .
$$

We interpret this total transition probability $P_{i}(t)$ as the degree of decoherence caused by spin-flip processes over time $t$. Now, $|\langle k|V| i\rangle|^{2}=A^{2}\left[I(I+1)-I_{z}^{(k)}\left(I_{z}^{(k)}+1\right)\right] / 4$. Assuming some distribution of the nuclear spins we can replace this matrix element by its average value (denoted by brackets) where $\sqrt{\left\langle\left(I_{z}^{(k)}\right)^{2}\right\rangle}$ describes then the variance of the mean value $\left\langle I_{z}^{(k)}\right\rangle=p I$. E.g. a Poissonian distribution gives $|\langle k|V| i\rangle|^{2} \approx A^{2}[I(I+1)-p I(p I+1)] / 4$, in which case the matrix element vanishes for full polarization parallel to the electron-spin (i.e. $p=1$ ), as required by conservation of total spin. $P_{i}(t)$ is strongly suppressed for final states for which $t_{0} \equiv 2 \pi \hbar /\left|E_{i}-E_{f}\right| \ll t$, which simply reflects conservation of energy. In particular, for a substantial nuclear polarization, i.e. $p^{2} N \gg 1, P_{i}(t)$ oscillates in time but with the vanishingly small amplitude $1 / p^{2} N$ (for $B=0$ ). We can estimate $N$ to be on the order of the number of atoms per quantum dot, which is about $10^{5}$. Such a situation with $p^{2} N \gg 1$ can be established by dynamically spin-polarizing the nuclear spins (Overhauser effect) e.g. via optical pumping [38 or via spinpolarized currents at the edge of a 2DEG [39]. This gives rise to an effective nuclear field $B_{\mathrm{n}}=A p N I / g \mu_{\mathrm{B}}$ which is reported to be as large as $B_{\mathrm{n}}^{*}=4 \mathrm{~T}$ in GaAs (corresponding to $p=0.85$ ) [39] and which has a lifetime on the order of minutes [38]. Alternatively, for unpolarized nuclei with $p=0$ but a field $B$ in the Tesla range, the amplitude of $P_{i}(t)$ vanishes as $\left(A I N / g \mu_{\mathrm{B}} B\right)^{2} / N \approx\left(B_{\mathrm{n}}^{*} / B\right)^{2} / N \ll 1$. For $B$ or $B_{\mathrm{n}}=1 \mathrm{~T}$ the oscillation frequency $1 / t_{0}$ of $P_{i}(t)$ is about $10 \mathrm{GHz}$. Thus, spin flip processes and hence dephasing due to nuclear spins can be strongly suppressed, either by dynamically polarizing the nuclear spins and/or by applying a magnetic field $B$. The remaining dephasing effects (described again by a weak-coupling master equation [11]) should then be small enough to be eliminated by error correction.

We now address the imperfections of the quantum gate operation. For this we note first that, for the purpose of quantum computing, the qubits must be coupled only for the short time of switching $\tau_{s}$, while most of the time there is to be no coupling between the dots. We estimate now how small we can choose $\tau_{s}$. For this we consider a scenario where $J$ (initially zero) is adiabatically switched on and off again during the time $\tau_{s}$, e.g. by an electrical gate by which we lower and then raise again the barrier $V(t)$ between the dots (alternatively, we can vary $B$, $a$, or $E$ ). A typical frequency scale during switching is given by the exchange energy (which results in the coherent tunneling between the dots) averaged over the time interval of switching, $\bar{J}=\left(1 / \tau_{s}\right) \int_{0}^{\tau_{s}} d t J(t)$. Adiabaticity then requires that many coherent oscillations (characterized approximately by $\bar{J}$ ) have to take place in the double-well system while the control parameter $v=$ $V, B, a$, or $E$ is being changed, i.e. $1 / \tau_{s} \approx|\dot{v} / v| \ll \bar{J} / \hbar$. If this criterion is met, we can use our equilibrium analysis to calculate $J(v)$ and then simply replace $J(v)$ by $J(v(t))$ in case of a time-dependent control parameter $v(t)$ 41. Note that this is compatible with the requirement needed for the XOR operation, $J \tau_{s} / \hbar=n \pi, \mathrm{n}$ odd, if we choose $n \gg 1$. Our method of calculating $J$ is self-consistent if $J \ll \Delta \epsilon$, where $\Delta \epsilon$ denotes the single-particle level spacing. The combination of both inequalities yields $1 / \tau_{s} \ll \bar{J} / \hbar \ll \Delta \epsilon / \hbar$, i.e. no higherlying levels can be excited during the switching. Finally, since typically $J \approx 0.2 \mathrm{meV}$ we see that $\tau_{s}$ should not be smaller than about $50 \mathrm{ps}$. Now, during the time $\tau_{s}$ spin and charge couple and thus dephasing in the charge sector described by $\tau_{\phi}^{c}$ can induce dephasing of spin via an uncontrolled fluctuation $\delta J$ of the exchange coupling. However, this effect is again small, it can be estimated to be on the order of $\tau_{s} / \tau_{\phi}^{c} \sim 10^{-2}$, since even for large $\operatorname{dots} \tau_{\phi}^{c}$ is reported to be on the order of nanoseconds [24]. This seems to be a rather conservative estimate and one can expect the spin dephasing to be considerably smaller since not every charge dephasing event will affect the spin. Finally, weak dephasing of the effective spin Hamiltonian during switching has been described elsewhere [1] in terms of a weak-coupling master equation which accounts explicitly for decoherence of the spins during the 
switching process. Based on this analysis [11], the probability for a gate error per gate operation (described by $\mathcal{K}_{2}$ in Eq. (13) of [11]) is estimated to be approximately $\tau_{s} / \tau_{\phi}^{c} \sim 10^{-2}$ or better (see above).

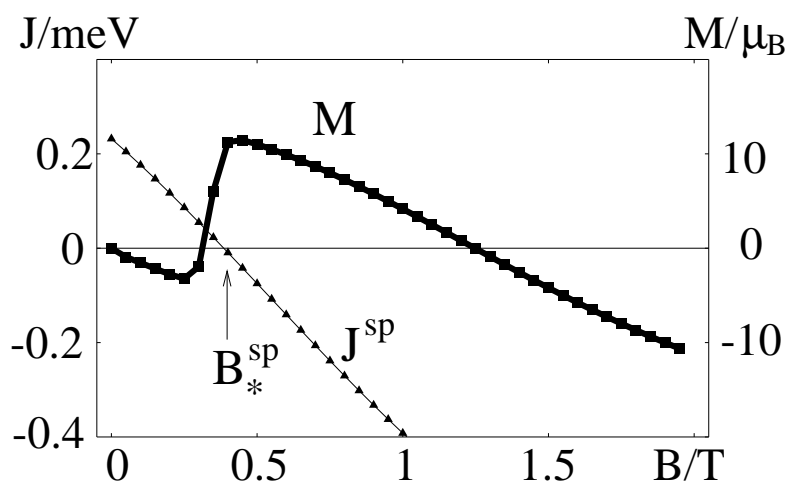

FIG. 4. The equilibrium magnetization $M$ (box-shaped symbols) in units of Bohr magnetons $\mu_{\mathrm{B}}$ as a function of magnetic field. $M$ is obtained numerically from the sp-hybridized Heitler-London approximation. Note that the magnetization exhibits a jump at the field value $B_{*}^{\text {sp }}$ for which the exchange $J^{\text {sp }}$ (triangle symbols) changes sign. At the left and right hand side of the jump the negative slope of $M(B)$ indicates orbital diamagnetism. The temperature for this plot is $T=0.2 \mathrm{~K}$, while as before $\hbar \omega_{0}=3 \mathrm{meV}$ and $a=0.7 a_{\mathrm{B}}$.

\section{EXPERIMENTAL IMPLICATIONS}

Coherent coupling between the states of neighboring dots is the keystone of our proposal for quantum gate operation, and experimental probes of this coupling will be very interesting to explore. The effect of the dot-dot coupling manifests itself in the level structure, which could be measured non-invasively with spectroscopic methods [3, 1. An alternative way is to measure the static magnetization in response to a magnetic field $B$ which is applied along the $z$-axis. This equilibrium magnetization is given by $M=g \mu_{\mathrm{B}} \operatorname{Tr}\left(S_{1}^{z}+S_{2}^{z}\right) e^{-\left(H_{s}+H_{Z}\right) / k T}$, where $H_{s}$ is given in Eq. (11), and $H_{\mathrm{Z}}=g \mu_{\mathrm{B}} \sum_{i} \mathbf{B}_{i} \cdot \mathbf{S}_{i}$ is the Zeeman term. It is straightforward to evaluate $M$, and in Fig. 1 we plot $M$ as a function of $B$ for a typical temperature $T=0.2 \mathrm{~K}$. The exchange $J^{\mathrm{sp}}(B)$ is also shown in Fig. 4 . Both $J^{\mathrm{sp}}(B)$ and $M$ are the results of the sp-hybridized Heitler-London approximation. We note that the equilibrium magnetization $M(B)$ is strongly dominated by the orbital response (via the exchange $J$ ); we find a diamagnetic response (negative slope of $M$ ) for $B<B_{*}^{\text {sp }}$ which is followed by a pronounced jump in the magnetization at the field $B_{*}^{\text {sp }}$ followed again by a diamagnetic response. Experimental observation of this jump would give evidence for the existence of the predicted singlet-triplet level-crossing at $B_{*}^{\mathrm{sp}}$, and such measurements would allow one to "map out" $J$ around the point where it can be tuned to zero, e.g. by also varying the barrier between the dots. The magnetic moment produced by the orbital motion of the electrons in one pair of coupled quantum dots at the peak $\left(B=B_{*}^{\mathrm{sp}}\right)$ is around $10 \mu_{\mathrm{B}}$ (see Fig. (4). This signal could be further amplified by using an ensemble of pairs of coupled quantum dots.

A further way to get experimental information about the exchange coupling would be to measure the spin response to an ac magnetic field (in the linear-response regime), described by the dynamical spin susceptibilities $\chi_{m n}^{p q}(\omega)=(i / \hbar) \int_{0}^{\infty} d t \exp (i \omega t)\left\langle\left[S_{m}^{p}(t), S_{n}^{q}(0)\right]\right\rangle$, where $m, n=1,2$, and $p, q=x, y, z$. Being interested in the spin response only, we assume this ac field to be applied in plane so that there is no orbital response (for a sufficiently weak field with no subband mixing). We see then that all the transverse spin susceptibilities $\chi_{m n}^{p \neq q, q}$ vanish, and we are left with the longitudinal ones only, where $\chi_{m n}^{x x}=$ $\chi_{m n}^{y y}=\chi_{m n}^{z z} \equiv \chi_{m n}$ due to the rotational symmetry of $H_{\mathrm{s}}$. It is sufficient to consider the dissipative part, $\chi_{m n}^{\prime \prime}(\omega)=$ $\operatorname{Im} \chi_{m n}(\omega)$, for which we obtain $\chi_{11}^{\prime \prime}=\chi_{22}^{\prime \prime}=-\chi_{12}^{\prime \prime}=$ $-\chi_{21}^{\prime \prime}=-(\pi / 4) f(J, B)[\delta(\hbar \omega+J)-\delta(\hbar \omega-J)]$, where $f(J, B)=\left(e^{J / k T}-1\right) /\left(1+e^{J / k T}+2 \cosh \left(g \mu_{\mathrm{B}} B / k T\right)\right)$. Also, due to conservation of total spin, the total response, $\chi_{1 j}+\chi_{2 j}$, as well as the response to a spatially uniform field, $\chi_{i 1}+\chi_{i 2}$, vanish. Thus, to observe the spin susceptibilities calculated here one needs to apply the fields locally or to measure the spin of a dot separately; both cases could be realized e.g. by atomic or magnetic force microscopes (see also below, where we briefly discuss local fields produced by field gradients).

\section{CONCLUDING REMARKS}

We end with a few comments on a network of coupled quantum dots in the presence of fields (see also Ref. [11). In a set-up with only one quantum gate (i.e. two quantum dots) the gate operation can be performed using uniform magnetic fields (besides electric gates), while in a quantum computer with many gates, which have to be controlled individually, local magnetic fields are indispensable, especially for the single-qubit gates [11,42]. However, we emphasize that it is not necessary that every single quantum dot in a network is directly addressable with a local magnetic field. Indeed, using "swap" operations $U_{\mathrm{sw}}$, any qubit-state can be transported to a region where the single-qubit gate operation is performed, and then back to its original location, without disturbing this or other qubits. In one possible mode of operation a constant field $B_{*}$, defined by $J\left(B_{*}\right)=0$, is applied, while smaller time-dependent local fields then control the gate operations. We can envision local fields being achieved by a large number of techniques: with neighboring magnetic dots [11], closure domains, a grid of current-carrying wires below the dots, tips of magnetic or atomic force microscopes, or by bringing the qubit into contact (by shifting the dot via electrical gating) with a region containing magnetic moments or nuclear 
spins with different hyperfine coupling (e.g. AlGaAs instead of GaAs)-and others. A related possibility would be to use magnetic field gradients. Single-qubit switching times of the order of $\tau_{s} \approx 20 \mathrm{ps}$ require a field of $1 \mathrm{~T}$, and for an inter-dot distance $2 a \approx 30 \mathrm{~nm}$, we would need gradients of about $1 \mathrm{~T} / 30 \mathrm{~nm}$, which could be produced with commercial disk reading/writing heads. [The operation of several XOR gates via magnetic fields also requires gradients of similar magnitude.] Alternatively, one could use an ac magnetic field $B_{\mathrm{ac}}$ and apply electron spin resonance (ESR) techniques to rotate spins with a single-qubit switching time (at resonance) $\tau_{s} \approx \pi \hbar / B_{\text {ac }}$. To address the dots of an array individually with ESR, a magnetic field gradient is needed which can be estimated as follows. Assuming a relative ESR linewidth of $1 \%$ and again $2 a=30 \mathrm{~nm}$ we find about $B_{\mathrm{ac}} \cdot 10^{4} \mathrm{~cm}^{-1}$. Field gradients in excitation sequences for NMR up to $2 \cdot 10^{4} \mathrm{G} / \mathrm{cm}$ have been generated [40] which allows for $B_{\mathrm{ac}} \approx 1 \mathrm{G}$. The resulting switching times, however, are rather long, on the order of $100 \mathrm{~ns}$, and larger field gradients would be desirable. Finally, such ESR techniques could be employed to obtain information about the effective exchange values $J$ : the exchange coupling between the spins leads to a shift in the spin resonance frequency which we found to be of the order of $J / \hbar$ by numerical analysis 34.

To conclude, we have calculated the exchange energy $J(B, E, a)$ between spins of coupled quantum dots (containing one electron each) as a function of magnetic and electric fields and inter-dot distance using the HeitlerLondon, hybridized Heitler-London, and Hund-Mulliken variational approach. We have shown that $J(B, E, a)$ changes sign (reflecting a singlet-triplet crossing) with increasing $B$ field before it vanishes exponentially. Besides being of fundamental interest, this dependence opens up the possibility to use coupled quantum dots as quantum gate devices which can be operated by magnetic fields and/or electric gates (between the dots) to produce entanglement of qubits.

\section{ACKNOWLEDGMENTS}

We would like to thank J. Kyriakidis, S. Shtrikman, and E. Sukhorukov for useful discussions. This work has been supported in part by the Swiss National Science Foundation.

\section{APPENDIX A: HUND-MULLIKEN MATRIX ELEMENTS}

Here, we list the explicit expressions for the matrix elements defined in Eqs. (9) and (10) as a function of the dimensionless inter-dot distance $d=a / a_{\mathrm{B}}$ and the magnetic compression factor $b=\sqrt{1+\omega_{\mathrm{L}}^{2} / \omega_{0}^{2}}$ where
$\omega_{\mathrm{L}}=e B / 2 m c$. The single-particle matrix elements are given by

$$
\begin{aligned}
\epsilon & =\frac{3}{32} \frac{1}{b^{2} d^{2}}+\frac{3}{8} \frac{S^{2}}{1-S^{2}}\left(\frac{1}{b}+d^{2}\right)+b, \\
t & =\frac{3}{8} \frac{S}{1-S^{2}}\left(\frac{1}{b}+d^{2}\right)
\end{aligned}
$$

where we used $S=\exp \left(-d^{2}(2 b-1 / b)\right.$ ). The (twoparticle) Coulomb matrix elements can be expressed as

$$
\begin{aligned}
V_{+} & =N^{4}\left(4 g^{2}\left(1+S^{2}\right) F_{1}+\left(1+g^{2}\right)^{2} F_{2}\right. \\
& \left.+4 g^{2} F_{3}-16 g^{2} F_{4}\right), \\
V_{-} & =N^{4}\left(1-g^{2}\right)^{2}\left(F_{2}-S^{2} F_{3}\right), \\
U & =N^{4}\left(\left(1+g^{4}+2 g^{2} S^{2}\right) F_{1}+2 g^{2} F_{2}\right. \\
& \left.+2 g^{2} S^{2} F_{3}-8 g^{2} F_{4}\right), \\
X & =N^{4}\left[\left(\left(1+g^{4}\right) S^{2}+2 g^{2}\right) F_{1}+2 g^{2} F_{2}\right. \\
& \left.+2 g^{2} S^{2} F_{3}-8 g^{2} F_{4}\right] \\
w & =N^{4}\left(-g\left(1+g^{2}\right)\left(1+S^{2}\right) F_{1}-g\left(1+g^{2}\right) F_{2}\right. \\
& \left.-g\left(1+g^{2}\right) S^{2} F_{3}+\left(1+6 g^{2}+g^{4}\right) S F_{4}\right),
\end{aligned}
$$

with $N=1 / \sqrt{1-2 S g+g^{2}}$ and $g=\left(1-\sqrt{1-S^{2}}\right) / S$. Here, we make use of the functions

$$
\begin{aligned}
F_{1} & =c \sqrt{b}, \\
F_{2} & =c \sqrt{b} e^{-b d^{2}} \mathrm{I}_{0}\left(b d^{2}\right), \\
F_{3} & =c \sqrt{b} e^{d^{2}(b-1 / b)} \mathrm{I}_{0}\left(d^{2}(b-1 / b)\right), \\
F_{4} & =c \sqrt{b} e^{-d^{2} / 4 b} \times \\
& \times \sum_{k=-\infty}^{\infty}(-1)^{k} \mathrm{I}_{2 k}\left(\frac{d^{2}}{4}(2 b-1 / b)\right) \mathrm{I}_{2 k}\left(i \frac{d^{2}}{2} \sqrt{b^{2}-1}\right),
\end{aligned}
$$

where $\mathrm{I}_{n}$ denotes the Bessel function of $n$-th order. For our purposes, we can neglect terms with $|k|>1$ in the sum in $F_{4}$, since for $\hbar \omega_{0}=3 \mathrm{meV}, B<30 \mathrm{~T}$, and $d=0.7$ the relative error introduced by doing so is less than $1 \%$.

* mail: burkard@ubaclu.unibas.ch

$\dagger \quad$ mail: loss@ubaclu.unibas.ch

¥ mail: divince@watson.ibm.com

[1] L. Jacak, P. Hawrylak, and A. Wójs, Quantum Dots (Springer, Berlin, 1997).

[2] L. P. Kouwenhoven, C. M. Marcus, P. L. McEuen, S. Tarucha, R. M. Westervelt, and N. S. Wingreen, Proceedings of the Advanced Study Institute on Mesoscopic Electron Transport, edited by L. L. Sohn, L. P. Kouwenhoven, G. Schön (Kluwer, 1997).

[3] R. C. Ashoori, Nature 379, 413 (1996). 
[4] R. J. Luyken, A. Lorke, M. Haslinger, B. T. Miller, M. Fricke, J. P. Kotthaus, G. Medeiros-Ribiero, and P. M. Petroff, preprint.

[5] S. Tarucha, D. G. Austing, T. Honda, R. J. van der Hage, and L. P. Kouwenhoven, Phys. Rev. Lett. 77, 3613 (1996); L. P. Kouwenhoven, T. H. Oosterkamp, M. W. S. Danoesastro, M. Eto, D. G. Austing, T. Honda, and S. Tarucha, Science 278, 1788 (1997).

[6] F. R. Waugh, M. J. Berry, D. J. Mar, R. M. Westervelt, K. L. Chapman, and A. C. Gossard, Phys. Rev. Lett. 75, 705 (1995); C. Livermore, C. H. Crouch, R. M. Westervelt, K. L. Chapman, and A. C. Gossard, Science 274, 1332 (1996).

[7] T. H. Oosterkamp, S. F. Godijn, M. J. Uilenreef, Y. V. Nazarov, N. C. van der Vaart, and L. P. Kouwenhoven, Phys. Rev. Lett. 80, 4951 (1998).

[8] R. H. Blick, D. Pfannkuche, R. J. Haug, K. v. Klitzing, and K. Eberl, Phys. Rev. Lett. 80, 4032 (1998); R. H. Blick, D. W. van der Weide, R. J. Haug, K. Eberl, Phys. Rev. Lett. 81, 689 (1998).

[9] K. Nomoto, R. Ugaijn, T. Suzuki, and I. Hase, J. Appl. Phys. 79, 291 (1996); A. O. Orlov, I. Amlani, G. H. Bernstein, C. S. Lent, and G. L. Snider, Science 277, 928 (1997).

[10] D. Deutsch, Proc. R. Soc. Lond. A 400, 97 (1985).

[11] D. Loss and D. P. DiVincenzo, Phys. Rev. A 57, 120 (1998).

[12] I. L. Chuang, N. A. Gershenfeld, and M. Kubinec, Phys. Rev. Lett. 80, 3408 (1998).

[13] D. Cory, A. Fahmy, and T. Havel, Proc. Nat. Acad. Sci. U.S.A. 94, 1634 (1997).

[14] J. A. Jones, M. Mosca, R. H. Hansen, Nature 393, 344 (1998).

[15] J. I. Cirac and P. Zoller, Phys. Rev. Lett. 74, 4091 (1995); C. Monroe, D. M. Meekhof, B. E. King, W. M. Itano, and D. J. Wineland, Phys. Rev. Lett. 75, 4714 (1995).

[16] Q. A. Turchette, C. J. Hood, W. Lange, H. Mabuchi, and H. J. Kimble, Phys. Rev. Lett. 75, 4710 (1995).

[17] D. V. Averin, Solid State Commun. 105, 659 (1998); A. Shnirman, G. Schön, and Z. Hermon, Phys. Rev. Lett. 79, 2371 (1997).

[18] A. Barenco, D. Deutsch, A. Ekert, and R. Josza, Phys. Rev. Lett. 74, 4083 (1995).

[19] R. Landauer, Science 272, 1914 (1996).

[20] J. A. Brum and P. Hawrylak, Superlattices and Microstructures 22, 431 (1997).

[21] P. Zanardi and F. Rossi, quant-ph/9804016.

[22] Such leakage can happen e.g. in the switching process of single qubits or by coupling two qubits together etc. and can easily lead to uncontrollable errors. This concern is especially relevant in quantum dots where the energy level spacing is (nearly) uniform (in contrast to real atoms) so that the levels defining the qubit are of similar scale as the separation to neighboring energy levels.

[23] J. M. Kikkawa and D. D. Awschalom, Phys. Rev. Lett. 80, 4313 (1998).

[24] A. G. Huibers, M. Switkes, C. M. Marcus, K. Campman, and A. C. Gossard, Phys. Rev. Lett. 81, 200 (1998).

[25] The dephasing times of Refs. 23 24 are both measured in GaAs semiconductors which involve many electrons.
It would be highly desirable to get direct experimental information about dephasing times in isolated quantum dots of low filling as considered here.

[26] D. P. DiVincenzo, Phys. Rev. A 51, 1015 (1995).

[27] A. Barenco, C. H. Bennett, R. Cleve, D. P. DiVincenzo, N. Margolus, P. Shor, T. Sleator, J. A. Smolin, and H. Weinfurter, Phys. Rev. A 52, 3457 (1995).

[28] B. E. Kane, Nature 393, 133 (1998).

[29] D.C. Mattis, The Theory of Magnetism, Vol. I, Sec. 4.5, Springer Series in Solid-State Sciences 17 (Springer, New York, 1988).

[30] D. P. DiVincenzo and D. Loss, Superlattices and Microstructures 23, 419 (1998).

[31] V. Fock, Z. Phys. 47, 446 (1928); C. Darwin, Math. Proc. Cambridge Phil. Soc. 27, 86 (1930).

[32] M. Wagner, U. Merkt, and A.V. Chaplik, Phys. Rev. B 45, 1951 (1992).

[33] D. Pfannkuche, V. Gudmundsson, and P. A. Maksym, Phys. Rev. B 47, 2244 (1993).

[34] G. Burkard, D. Loss, and D. P. DiVincenzo, unpublished.

[35] See e.g. E. Fradkin, Field Theories of Condensed Matter Systems (Addison-Wesley, 1991).

[36] We note that the significant changes due to Coulomb long-range interactions are valid down to the scale of real atoms. Since atomic orbitals and the harmonic orbitals used here behave similarly (for $B=0$ ), we expect to find qualitatively similar results for real molecules (as found here for coupled dots) especially as regards the effect of Coulomb long-range interactions on $t_{\mathrm{H}}, U_{\mathrm{H}}, J$ and their dependence on the inter-atomic distance $a$.

[37] J. Preskill, quant-ph/9712048.

[38] M. Dobers, K. v. Klitzing , J. Schneider, G. Weimann, and K. Ploog, Phys. Rev. Lett. 61, 1650 (1988).

[39] D. C. Dixon, K. R. Wald, P. L. McEuen, and M. R. Melloch, Phys. Rev. B 56, 4743 (1997).

[40] D. Cory and W. Zhang, Phys. Rev. Lett. 80, 1324 (1998).

[41] If during the change of $v(t)$ the total spin remains conserved, no transitions between the instantaneous singlet and triplet eigenstates can be induced during the switching. Thus, the singlet and triplet states evolve independently of each other, and the condition on adiabatic switching involves $\Delta \epsilon$ (instead of $J$ ), i.e. we only need to require that $1 / \tau_{s} \approx|\dot{v} / v| \ll \overline{\Delta \epsilon} / \hbar$, which would be less restrictive. Also, only $\int_{0}^{\tau_{s}} d t J(t)$ and not $J(t)$ itself is needed for the gate operation. Therefore, the adiabaticity criterion given in the text, while being sufficient, need not be really necessary. However, the complete analysis of the time-dependent problem in terms of variational wave functions is beyond the scope of the present work and will be addressed elsewhere.

[42] We note that it is sufficient to have single-qubit rotations about any two orthogonal axes. A preferable choice here are two orthogonal in-plane axes because magnetic fields $\mathbf{B}_{\|}$parallel to the 2DEG do not affect the exchange coupling $J\left(B_{\perp}\right)$ (assuming that we can exclude subband mixing induced by a sufficiently strong $B_{\|}$). 